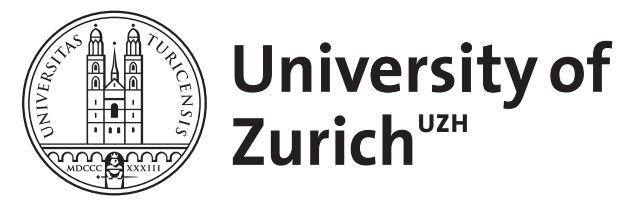

\title{
Quality control requirements in neonatal screening
}

\author{
Torresani, Toni
}

\begin{abstract}
One of the key tasks for the persons in charge of a neonatal screening laboratory is the control and maintenance of the quality of analytical results. The process of examining every day hundreds of blood spot samples obtained from newborn babies in order to divide the population of neonates into a low risk group and a high risk group, presents several challenges with respect to organisation, analytical accuracy and precision and effectiveness in the recall and treatment procedures. Quality in neonatal screening is not determined solely by the analytical performance of the laboratory; all the steps of the neonatal screening "system" contribute to the overall quality and performance. Conclusion: programmes for the monitoring of the quality of neonatal screening have to be multidisciplinary and not restricted to the laboratory alone; not only analytical performance, but also the procedures used throughout the programme have to be monitored
\end{abstract}

DOI: https://doi.org/10.1007/s00431-003-1353-1

Posted at the Zurich Open Repository and Archive, University of Zurich

ZORA URL: https://doi.org/10.5167/uzh-156047

Journal Article

Published Version

Originally published at:

Torresani, Toni (2003). Quality control requirements in neonatal screening. European Journal of Pediatrics, 162:S54-S56.

DOI: https://doi.org/10.1007/s00431-003-1353-1 


\section{Quality control requirements in neonatal screening}

Published online: 28 November 2003

(C) Springer-Verlag 2003

\begin{abstract}
One of the key tasks for the persons in charge of a neonatal screening laboratory is the control and maintenance of the quality of analytical results. The process of examining every day hundreds of blood spot samples obtained from newborn babies in order to divide the population of neonates into a low risk group and a high risk group, presents several challenges with respect to organisation, analytical accuracy and precision and effectiveness in the recall and treatment procedures. Quality in neonatal screening is not determined solely by the analytical performance of the laboratory; all the steps of the neonatal screening "system" contribute to the overall quality and performance. Conclusion: Programmes for the monitoring of the quality of neonatal screening have to be multidisciplinary and not restricted to the laboratory alone; not only analytical performance, but also the procedures used throughout the programme have to be monitored.
\end{abstract}

Keywords Neonatal screening · Newborn screening · Quality assurance $\cdot$ Quality control

Abbreviations $N B S$ newborn screening $~ Q A$ quality assurance $\cdot Q C$ quality control

\section{Introduction}

Quality, quality control (QC) and quality assurance (QA) are very frequently used words in today's healthcare environment. Even if the definitions of these words are universal, their application in everyday life must be customised to the task involved.

\footnotetext{
T. Torresani

Department of Endocrinology and Diabetology,

University Children's Hospital Zürich,

Steinwiesstrasse 75 ,

8032 Zürich, Switzerland

E-mail: Toni.Torresani@kispi.unizh.ch

Tel.: + 41-1-2667111

Fax: + 41-1-2667166
}

For newborn screening (NBS), a public health activity aimed at the early presymptomatic identification of infants who are affected by certain genetic, metabolic or infectious conditions, these requirements are particularly challenging. As one of the best-accepted procedures in preventive paediatric medicine, NBS is the result of a complex collaborative process. It involves medical professions, authorities, professional and parent organisations. All these individuals and interest groups have to collaborate closely to achieve the goals of NBS. Table 1 summarises the main components of the NBS "system" and the means used to establish and monitor quality.

Success or failure, consequently quality, is in such a process extremely dependent upon the performance of the weakest component of the NBS "system". Thus, quality is not a product of coincidence but a wellplanned exercise.

When establishing a NBS programme, the aims to be achieved must be clearly defined, taking into account the local infrastructure and the social and political environment. These definitions will determine and influence the final outcome of the programme. Careful planning will help enhance quality; the "obsession" to design a programme aimed at detecting each and every case of a certain disease, as unfortunately seen in many NBS programmes worldwide, will most probably result in poor quality.

\section{Information and education}

In the world of NBS, a central role in the planning of quality is occupied by information and education. A programme for NBS must be known and accepted in the general population. This will greatly increase the likelihood that no newborn escapes the sample collection; thus information and educational activities aimed at the families and the general public, for example as information brochures or radio and TV spots, have proven to be a good communication vehicle. At the same time, 
Table 1 Main components of NBS and measures taken to achieve quality

\begin{tabular}{ll}
\hline NBS component: & QC/QA achieved by: \\
\hline Sample collection & Education, information and logistics \\
Sample transport & Logistics \\
Sample analysis & Methodology and logistics \\
Recall of affected babies & Education, information and logistics \\
Treatment and follow-up & Education and information \\
\hline
\end{tabular}

NBS must be accepted by the medical professions. By preparing disease-related guidelines for NBS programmes, as done for example by the European Society for Paediatric Endocrinology in the case of congenital hypothyroidism and congenital adrenal hyperplasia $[1,2]$, clear explanations of the aims of the NBS program can be given and accordingly, rules can be set.

A critical point in NBS is the correct sample collection. In order to be suitable for NBS, a sample has to be collected at the right time and with the appropriate technique, the blood spotted on the designated and correctly labelled filter paper and all demographic information on the sample card has to be filled in correctly. Experience shows that all these goals can only be achieved by regular teaching the staff collecting the samples; particular attention here is required in explaining the reasons why the samples have to be collected in a certain way and during a specific time frame after birth.

A screening programme can only be successful if the babies identified by the system are subjected to timely and appropriate therapy. Therefore guidance on therapeutic measures and management of affected babies takes an important place in the establishment and maintenance of quality.

Finally every NBS programme should regularly be audited to check whether the established goals of the programme have been achieved. It has been shown that the publication of such information has a very beneficial effect on the overall quality.

\section{Logistics and laboratory methodology}

The core of a NBS programme is represented by the analytical procedures. It is therefore evident that the logistics of the laboratory and the analytical methodology take up a major role in the quality planning. Some of the key requirements for good quality are:

A. Laboratories should be planned to be able to handle a sufficiently large number of samples in order to build a significant base of experience. This translates into a number of samples large enough to provide a regular number of positive and borderline cases, i.e. one real case every $2-4$ weeks; and the samples should always be assayed on the same day they arrive in the laboratory. The major consequences arising from a too small number of samples are:
1. An inefficient screening due to poor cost benefit ratio and insufficient quality control.

2. Difficulties in the correct interpretation of results, particularly grey-zone or borderline results, due to little experience and insufficient statistical data

3. Excessively high number of repeat tests!

B. Care must be taken that the analytical methods used are:

1. Adequate to reliably detect the disease tested: an analytical method for NBS has to be reliable at the first analysis. Quite often sufficient material for a repeat test will not be available.

2. Adapted to the local infrastructure: analytical methods used in NBS differ significantly from country to country due to the differences in know how, available resources, financial possibilities, social and economical factors. The final result should remain the same.

3. Remember: quality has its price: low-cost reagents are not always the most economical. Excessive savings on the reagents and instrument side are in our experience usually coupled with high recall and/or repeat rates, frequent instrument breakdowns and as a consequence higher total costs. The final outcome will always be: poor overall quality of the programme.

C. The laboratory must implement an adequate level of internal quality control and participate in at least one external quality control scheme. The requirements for internal QC/QA should be planned in a way to assure the highest possible precision, the best rate of analytical error detection and the least number of samples repeated and/or rejected. Typically for QC monitoring, all assays where the internal QC samples are outside the \pm 2 SD limits are rejected. This rule is in our opinion too strict and will result in unnecessary assay rejections. In fact with a $\pm 2 \mathrm{SD}$ rule, $5 \%$ of the assays will be rejected even when everything is otherwise in order. Today there are several software packages available which facilitate the planning of analytical quality based on the real performance characteristics of the analytical method used [3]. With such software, stricter and statistically more viable rejection limits can be defined, resulting in an overall better analytical performance. The key to good results is the precision, sensitivity and specificity of the assay used.

\section{General considerations}

A score of initiatives aimed at improving all aspects of quality in NBS are underway internationally: standardisation of the filter paper used for sampling, guidelines to the choice of the "ideal" time for the collection of the screening sample, definition and use of calibrators and reference preparations, flow-sheets for 
recall procedures, guidelines for treatment procedures and for auditing of the screening system.

Even if numerous national and international organisations are involved in these initiatives, the major steps for obtaining and maintaining a high quality standard have to be undertaken at the level of every single programme. It is the work at the local level that mostly influences the outcome of neonatal screening and allows to achieve and maintain the level of quality necessary for an effective performance, all for the benefit of the affected babies detected through screening.

\section{Conclusions}

Quality in NBS, and not only in NBS, is a "cultural problem". Quality cannot be established and maintained simply by following blindly a set of rules; quality is an attitude and must be part of every day's life. Only if all individuals involved in screening take responsibility for their work, take pride in what they do and are highly motivated then there will be a substantial improvement in quality.

\section{References}

1. ESPE Working Group on Neonatal Screening (1999) Revised guidelines for neonatal screening programmes for primary congenital hypothyroidism. Horm Res 52: 49-52

2. ESPE Working Group on Neonatal Screening (2001) Procedure for neonatal screening for congenital adrenal hyperplasia due to 21-hydroxylase deficiency. Horm Res 55: 201-205

3. Suolinna E-M, Torresani T, Westgard JO (2201) Designing quality control for neonatal screening assays. http://www.westgard.com/qcapp19.htm 